Int. J. Dev. Biol. 63: 67-71 (2019)

https://doi.org/10.1387/ijdb.180312re

\title{
Evidence for an evolutionary relationship between Vmp1 and bacterial DedA proteins
}

\author{
LUIS-CARLOS TÁBARA, OLIVIER VINCENT and RICARDO ESCALANTE* \\ Instituto de Investigaciones Biomédicas Alberto Sols, CSIC, Universidad Autónoma de Madrid, Madrid, Spain
}

\begin{abstract}
VMP1 and DedA proteins are conserved families of transmembrane proteins in eukaryotes and prokaryotes respectively. Despite numerous reports involving these proteins in multiple cellular processes, their molecular function is still unknown. They share the domain of unknown function PF09335, suggesting a possible functional relationship between these protein families. Here we show that VMP1 from different species contain two short motifs conserved in the bacterial DedA proteins and the yeast protein Tvp38. The hallmark of one of these motifs is a glycine residue previously shown to be strictly conserved in all the DedA proteins. Substitution of this residue to leucine, glutamate or arginine in Dictyostelium Vmp1 inactivates the protein, as shown by the inability of the mutants to rescue the phenotypes associated with the lack of Vmp1 including development and lipid homeostasis. This is the first experimental approach that supports an evolutionary relationship between Vmp1 and DedA proteins and highlights the importance of the conserved glycine residue in the PF09335 domain.
\end{abstract}

KEY WORDS: autophagy, DedA, Dictyostelium, lipid homeostasis, Vmp1, PF09335, SNARE-associated Golgi protein

A proteomic analysis in $S$. cerevisiae identified several proteins present in a Golgi sub-compartment defined by the SNARE protein Tlg2. Among these proteins, Tvp38 (TIg2-compartment vesicle protein of $38 \mathrm{kDa}$ ) is a predicted transmembrane protein of unknown function (Inadome et al., 2005). A region of this protein was annotated as a pfam conserved domain (PF09335) and named "SNARE-associated Golgi protein". Despite the lack of an overall similarity, this domain is also detected in other eukaryotic proteins: VMP1, TMEM41A/B and TMEM64. Interestingly, the same domain is also found in a family of prokaryotic proteins, the so-called DedA proteins.

Prokaryotic DedA proteins are a family of conserved membrane proteins of unknown function initially identified in Escherichia coli. Phylogenetic analysis based on sequence homologies identified the presence of distantly related proteins of this family in bacteria, archaea and cyanobacteria (Boughner and Doerrler, 2012, Doerrler et al., 2013). They have been often denoted as the DedA/Tvp38 protein family to highlight the presence of the conserved PF09335 domains in these proteins (Keller et al., 2015, Keller and Schneider, 2013). DedA proteins are essential for cell viability and have been involved in a wide variety of functions, including cell division, membrane lipid composition, envelope stress responses and drug resistance (Boughner and Doerrler, 2012, Doerrler et al., 2013, Kumar and Doerrler, 2014, Ledgham et al., 2005, Sikdar et al., 2013).

The eukaryotic vacuole membrane protein 1 (VMP1) is now attracting much attention due to its important role in autophagy and ER homeostasis. VMP1 is a predicted multispanning membrane protein of unknown molecular function located in the endoplasmic reticulum (ER). VMP1 is highly conserved in most eukaryotes but is absent in the fungi lineage (Calvo-Garrido et al., 2008a). The effects of VMP1 deficiency have been studied in a number of model organisms and in cultured mammalian cells. Cells deficient in in this protein display pleiotropic defects in cell growth, osmoregulation, protein secretion, Golgi and ER structure, and autophagy in Dictyostelium discoideum (Calvo-Garrido et al., 2008a, Calvo-Garrido et al., 2008c, Calvo-Garrido and Escalante, 2010, Calvo-Garrido et al., 2014); cytokinesis, cell shape, structural defects of different organelles and alterations in the lipidic profile in Chlamydomonas reinhardtii (Tenenboim et al., 2014); autophagy and protein seAbbreviations used in this paper: ER, endoplasmic reticulum; TVP, TIg2-compartment
vesicle protein; VMP, vacuole membrane protein.

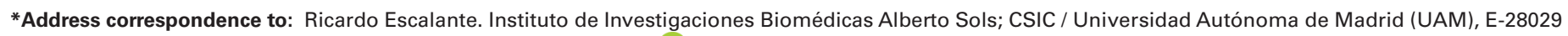
Madrid, Spain. Tel: +34 915854467. E-mail: rescalante@iib.uam.es - iD https://orcid.org/0000-0001-8547-531X
} 


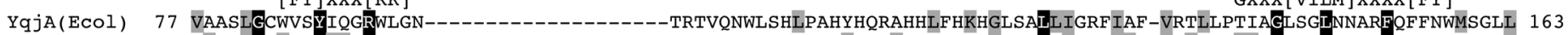

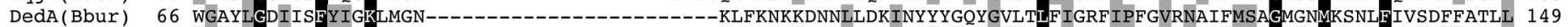

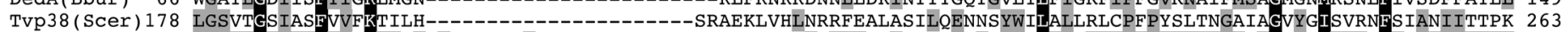

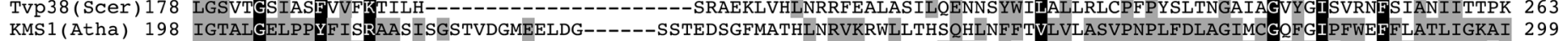

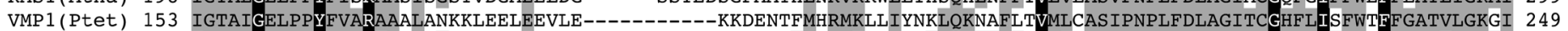

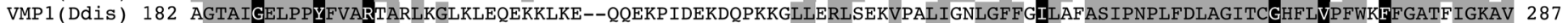

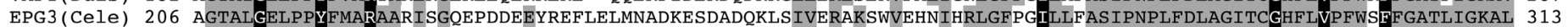

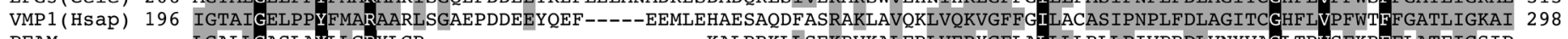
PFAM LGALGASLAYLLGRYLGR-----1

Fig. 1. Conservation of the PF09335 domain in DedA, Tvp38 and VMP1 proteins. The alignment includes amino acid sequences from YqjA (Ecol, E. coli), DedA (Bbur, B. burgdorferi), Tvp38 (Scer, S. cerevisiae), VMP1 from different organisms: Kms1 (Atha, Arabidopsis Thaliana), VMP1 (Ptet, P. tetraurelia; Ddis, D. discoideum; Hsap, H. sapiens), EPG3 (Cele, C. elegans), and the consensus for the PF09335 PFAM domain. Conserved (BLOSUM62) residues were shaded according to degree of conservation: black, >90\%; gray, 50-90\%. Numbers indicate amino acid positions and dashes indicate gaps. Shown above are consensus sequences of the two conserved motifs previously identified in the Tvp38/DedA family. Asterisk indicates the position of the glycine residue conserved in all DedA proteins.
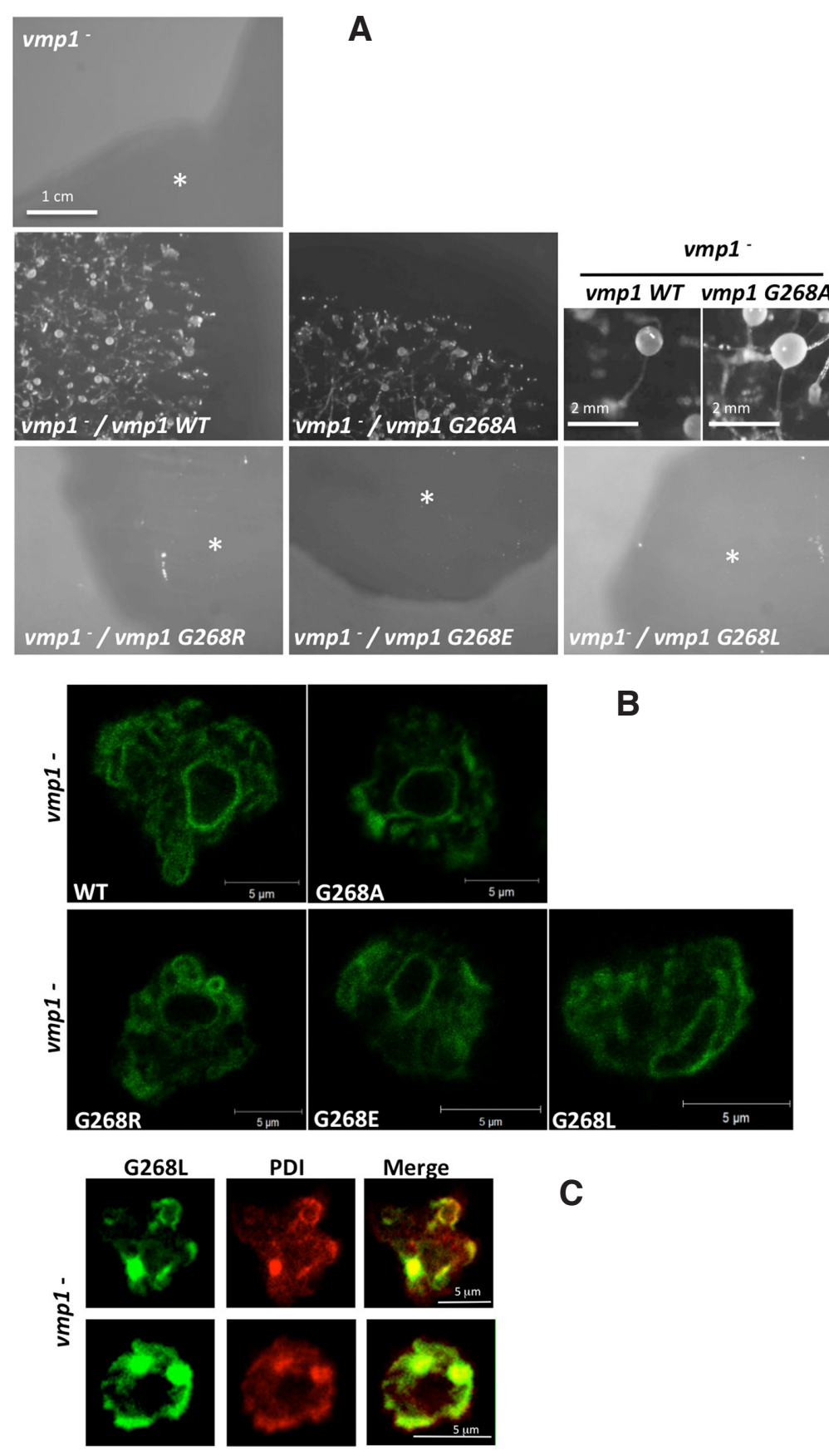

cretion defects in Caenorhabditis elegans and Drosophila melanogaster respectively (Bard et al., 2006, Tian et al., 2010); ER and Golgi structure defects in plants (Wang et al., 2011). Recent studies in mammalian cells provide an explanation for the complexity of these phenotypes as VMP1 has a role in the distribution and dynamics of ER subdomains enriched in lipid-synthesizing enzymes that are involved in a variety of membrane contact sites between the ER and other organelles (Tabara et al., 2018). VMP1 has been proposed to regulate lipid signalling at these contact sites and to play an essential role in cellular lipid and $\mathrm{Ca} 2+$ homeostasis (Tabara and Escalante, 2016, Tabara et al., 2018, Zhao et al., 2017). How VMP1 performs this function at the molecular level is still unknown.

The possibility of a functional relationship between eukaryotic VMP1 and prokaryotic DedA proteins is intriguing. Since the molecular function of VMP1 and DedA proteins is unknown, it is of great interest to investigate the functional conservation of the PF09335 domain in these proteins.

\section{Results and Discussion}

Previous sequence alignments of the PF09335 domain of DedA proteins and yeast Tvp38 revealed the presence of two short conserved motifs separated by variable length spacer sequences of approximately 50 amino acids. These motifs have the following consensus sequences: $F / Y x x x R / K$ and GxxxM/V/L/IxxxxF/Y (Keller and Schneider, 2013). The Gly in the second motif is the most conserved residue in all

Fig. 2. The conserved Gly residue of the PF09335 domain of Vmp1 is essential for Dictyostelium development. (A) vmp1 strain was transfected with expression plasmids carrying WT and mutated forms of Vmp1. After selection, transformants were analyzed in association with bacteria and representative pictures taken after 5 days. It should be noted that vmp1- cells are able to grow in association with bacteria forming a growth plaque (the dark zones labeled by asterisks) but are unable to develop under these conditions. Normal aggregation and fruiting body formation were only accomplished by the expression of WT and G268A Vmp 1 protein. Details of the fruiting bodies are displayed at the right. (B) Representative confocal images of cells expressing the different Vmp1 proteins. All proteins showed similar levels and localization. (C) Optical sections of two Dictyostelium vmp1- cells expressing the mutated Vmp1 G268L (green) and the colocalization with the ER membrane marker PDI (red). Some cells present accumulations of ER membrane. 
DedA proteins analyzed (Keller and Schneider, 2013). We have now performed sequence alignments of the predicted PF09335 domain of VMP1 and DedA proteins in different organisms, including the yeast protein Tvp38 (Fig. 1). Although the overall similarity between VMP1 and DedA proteins is very low, the two motifs of the PF09335 domain are very well conserved.

All tested VMP1 sequences from different species contain the conserved Gly residue, the only amino acid fully conserved in all DedA proteins (Keller and Schneider, 2013). It should be noted that the members of the TMEM41 and 64 families lack this Gly residue, which may indicate a higher divergence of these proteins with DedA proteins. In order to determine the functional importance of this residue, we used Dictyostelium discoideum since the inactivation of Vmp1 in this organism impairs its developmental stage and thus,

A
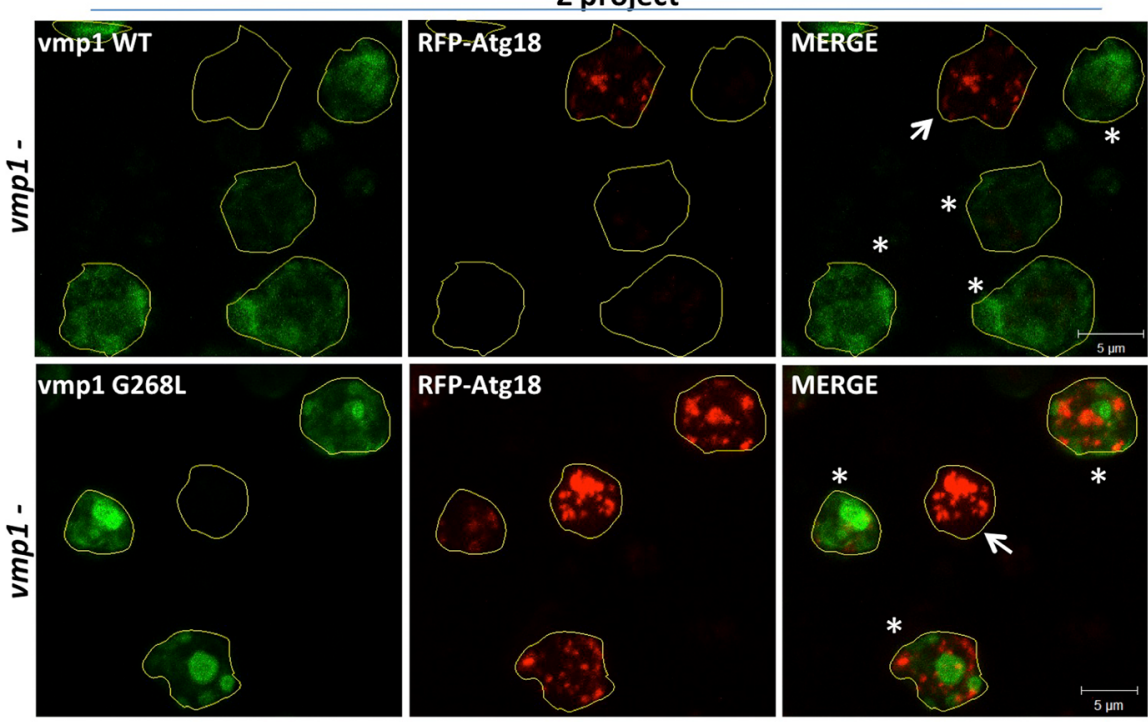

B
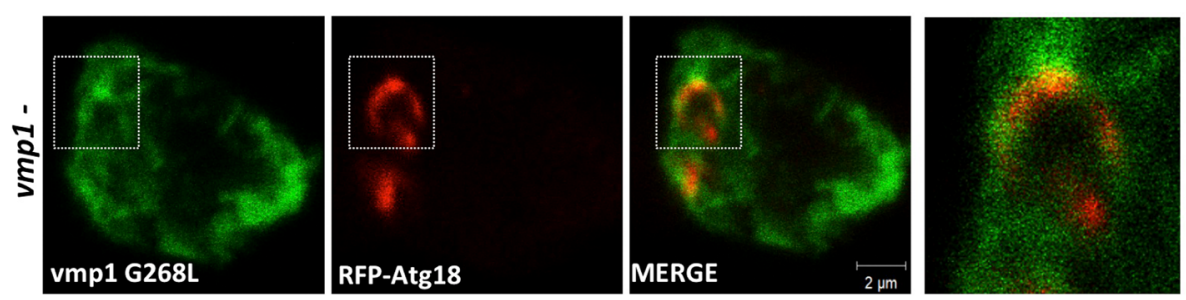

C
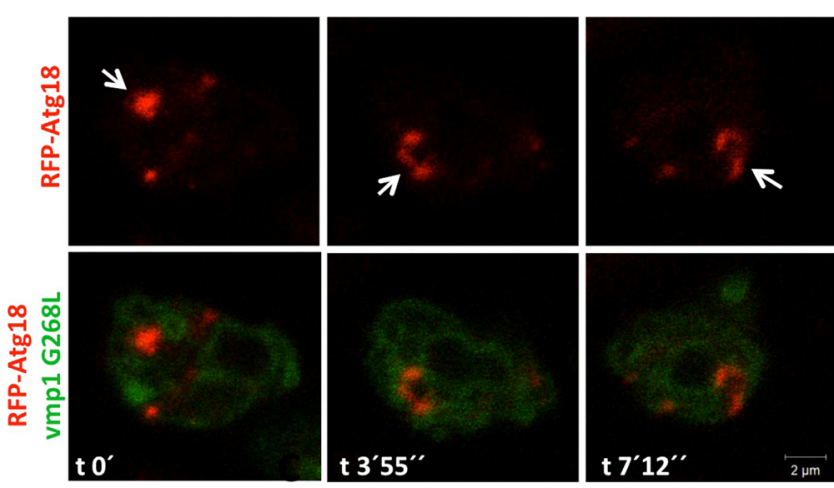

the functionality of the protein is relatively easy to assess. Besides developmental defects, Dictyostelium cells lacking Vmp1 are unable to grow in liquid media and have altered lipid homeostasis, as evidenced by the accumulation of the phospholipid Ptdlns3P in the ER(Calvo-Garrido et al., 2014). We generated point mutations in the Dictyostelium vmp1 gene to convert the conserved Gly $(\mathrm{G})$ residue at position 268 to the following amino acids: Ala (A), Leu (L), Arg (A) and Glu (E). The protein was fused to GFP at the C-terminus to determine its subcellular localization. The mutated constructs were transfected into Dictyostelium cells lacking vmp1 (Calvo-garrido et al., 2008b), and phenotypic rescue was evaluated. Both wild-type and G268A mutant were able to complement the growth in liquid media (data not shown) and develop into normal-looking fruiting bodies (Fig. 2A). In contrast, the other mutants containing the G268L, G268E and G268R substitutions were unable to complement the Vmp1-associated phenotypes, suggesting that the functionality of these mutant proteins is impaired (Fig. 2A). As shown in Fig. 2B, WT and mutant proteins showed similar levels of expression and similar localization at the ER, thus indicating that these mutations do not affect the stability or the correct subcellular localization of this protein. Interestingly, around $50 \%$ of non-rescued Vmp1 mutant cells showed accumulation of the Vmp1 signal in abnormal ER structures (confirmed by the colocalization with the ER marker PDI) (Fig. $2 \mathrm{C})$. This is in agreement with the defect in ER structure previously described in Dictyostelium and Arabidopsis Vmp1-null cells (Calvo-Garrido et al., 2008b, Wang et al., 2011).

It should be noted that the $E$. coli protein YqjA is, like Dictyostelium Vmp1, still functional when the conserved Gly residue is converted to Ala (Keller et al., 2015). Due to their small size and chemical properties, Gly and Ala seem to be interchangeable at least for the phenotypes we have examined. In contrast, a substitution to Leu, another non polar amino acid, or a conversion to a charged residue disrupt the functionality of Vmp1, suggesting

Fig. 3. Mutated Vmp1 is not able to rescue the homeostasis of Ptdlns3P. vmp1 cells expressing WT and G268L Vmp1 proteins fused to GFP were transfected with the Ptdlns3P marker RFP-Atg18 and visualized by confocal microscopy. (A) Maximum intensity projections of $x$-optical sections are shown. In the upper panel cells expressing WTVmp1 (green cells marked by asterisks) did not show the abnormal accumulation of RFP-Atg18 while the cell not expressing Vmp1 (marked by an arrow) show the accumulation of the marker. In the lower panel, cells show abnormal accumulation irrespective of the expression of the mutated form of Vmp1. (B) Colocalization analysis of Vmp1 G268L fused to GFP with Atg18 marker. A detail is shown on the right. (C) Different images at the times indicated from a confocal time lapse showing that the aberrant expression of the Atg18 marker is persistent and colocalizes with the mutated form of Vmp 1-GFP. 
that this conserved Gly residue and the corresponding short motif is important for Vmp1 function.

To determine whether the function of Vmp1 in lipid signalling is also impaired in the mutants, we analyzed the distribution of the autophagic protein Atg18, which interacts with the signaling lipid Ptdlns3P during autophagosome formation. It has been previously reported that Ptdlns3P is transiently generated in Dictyostelium WT cells but strongly accumulates in Vmp1 mutant cells (CalvoGarrido et al., 2014). The vmp1 null strain expressing either wildtype Vmp1 or the G268L mutant derivative were transformed with an expression marker encoding RFP-Atg18 and the fluorescence pattern was analyzed. As expected, expression of the WT form of Vmp1 results in a low RFP-Atg18 signal due to the rapid turnover of Ptdlns3P (Calvo-Garrido et al., 2014) and thus rescues the described aberrant accumulation of this marker (Fig. 3A). In contrast, expression of the protein containing the G268L substitution was unable to rescue the abnormal and characteristic accumulation of the Atg18 marker (Fig. 3A). Furthermore, this aberrant Ptdlns3P signaling event is associated with ER membranes containing the Vmp1 mutant form (Fig. 3B) and persists during long periods of times (Fig. 3C). All together, these results indicate that the G268L substitution leads to a loss of function of Dictyostelium Vmp1.

Although the molecular function of DedA proteins in bacteria remain elusive, it has been suggested that their function may be related to the stability of cellular membranes (Keller and Schneider, 2013; Sikdar et al., 2013). Interestingly, DedA mutants display defects in protein and solute transport across membranes and alterations in the proton motive force (Kumar and Doerrler, 2014, Kumar and Doerrler, 2015, Sikdar and Doerrler, 2010). It has been proposed that these defects could be primarily caused by alterations in the phospholipid composition of the cellular membrane (Doerrler et al., 2013, Sikdar et al., 2013, Thompkins et al., 2008). In fact, deletion of the unique DedA gene in Borrelia leads to decreased levels of phosphatidylethanolamine and increased levels of both phosphatidylglycerol and cardiolipin (Liang et al., 2010). Moreover, the major envelope stress response pathways that control the expression of genes involved in membrane integrity are strongly activated in DedA mutants (Sikdar et al., 2013).

VMP1 has been also involved in a wide variety of membranedependent processes (Calvo-garrido et al., 2008b, Calvo-Garrido et al., 2014, Tenenboim et al., 2014, Wang et al., 2011). In mammalian cells, VMP1 regulates the length of multiple membrane contact sites (Tabara and Escalante, 2016) and the dynamics of ER subdomains enriched in different phospholipid-synthesizing enzymes (Tabara et al., 2018). Moreover, metabolomics studies in Chlamydomonas demonstrated that the amount of phospholipid involved in membrane biogenesis decreases in VMP1-KO cells (Tenenboim et al., 2014). As in prokaryotes, a primary defect in membrane organization (the ER membrane in eukaryotes) may affect cellular lipid homeostasis, which would explain the pleiotropic defects in organelle biogenesis. In line with this hypothesis, the aberrant recruitment of the Ptdlns3P binding protein Atg18 that colocalize with the Vmp1 mutant isoforms strongly suggest that Vmp1 function could be related to the maintenance of a proper lipid signalling. Remarkably, it has been recently reported that the absence in mammalian cells of the VMP1-related protein TMEM41B (which also contains the PFAM09335 domain) leads to similar defects in both autophagosome and LD biogenesis to those observed after VMP1 depletion (Moretti et al., 2018, Morita et al., 2018). Furthermore it has been proposed that TMEM41B is also required for cellular lipid mobilization and distribution (Moretti et al., 2018).

In conclusion, our study provides experimental evidence for the functional conservation of the PF09335 domain and the essential role of a conserved Gly residue in Vmp1 and DedA proteins. Remarkably, the defective phenotypes associated with the absence or mutation of this domain in both bacteria and mammalian cells are linked to altered membrane homeostasis. The study of the function of this protein domain will be essential to decipher the molecular function of eukaryotic VMP1 and the prokaryotic DedA proteins.

\section{Materials and Methods}

\section{Dictyostelium growth and transformation}

All strains used in this work were derived from Dictyostelium discoideum AX4 strain. Dictyostelium cells were grown at $22^{\circ} \mathrm{C}$ axenically in HL5 liquid medium (Foremedium) supplemented with glucose $1 \%$ and penicillin-streptomycin (10 $000 \mathrm{U} / \mathrm{ml}$ penicillin and $10000 \mathrm{mg} / \mathrm{ml}$ streptomycin; Gibco). For growth in solid medium, Dictyostelium was seeded in association with a non pathogenic Klebsiella pneumoniae strain (Lima et al., 2018). Transformation were carried out by electroporation as previously described (Pang et al., 1999) and selected with the appropriate antibiotic. The mutant strain in Vmp1 was described previously (Calvo-Garrido et al., 2008c).

The plasmid RFP-Atg18 was kindly provided by Jason King (University of Sheffield).

\section{Site-directed mutagenesis}

Vmp1 coding sequence was cloned in a modified pBluescript SK between Spel and Bglll. $10 \mathrm{ng}$ of the plasmid was used as a template in a PCR reaction with a HiFi polymerase (KAPA biosystems) using overlapping oligonucleotides containing the desired mutations as described in the Stratagene QuickChange Site-Directed Mutagenesis protocol (https://www. genomics.agilent.com/files/Manual/200523.pdf). The mutation was verified by sequencing of the entire fragment. The mutated forms were cloned into pDM323 (Veltman et al., 2009).

\section{Confocal microscopy}

Cells were observed in vivo using lbidi $15 \mu$-Slide 8-well. For immunofluoresce cells were fixed with $2 \%$ paraformaldehyde in PBS $2 \mathrm{X}(1 \mathrm{X}$ PBS: $133 \mathrm{mM} \mathrm{NaCl}, 8 \mathrm{mM} \mathrm{Na}_{2} \mathrm{HPO}_{4}, 2 \mathrm{mM} \mathrm{KH}_{2} \mathrm{PO}_{4}$; $\mathrm{pH}$ 7.4) for $15 \mathrm{~min}$ at room temperature, washed 3 times for 5 min each with PBS $1 \mathrm{X}$ and then incubated with blocking buffer ( $2 \%$ BSA; $0.5 \%$ NP40 in PBS $1 \mathrm{X}$ ) for 1 hour. Cells were incubated overnight at $4^{\circ} \mathrm{C}$ with mouse monoclonal antibody against PDI (kindly provided by Dr. Pierre Cosson) at 1:1000 in blocking buffer. After three washes with PBS $1 \mathrm{X}$ cells were incubated for $1 \mathrm{~h}$ with the secondary antibody (1:500 in blocking buffer) conjugated to Alexa 546. After three washes with PBS $1 \mathrm{X}$ cells were mounted with Prolong for confocal visualization. Confocal images were acquired using an inverted Zeiss LSM 710 laser-scanning microscope.

\section{Acknowledgements}

This work has been supported by MINECO/FEDER (grant number BFU2015-64440-P). LC is supported by a fellowship from the Spanish "Ministerio de Educación Cultura y Deporte".

\section{References}

BARD, F., CASANO, L., MALLABiABARRENA, A., WALlACE, E., SAITO, K., KITAYAMA, H., GUIZZUNTI, G., HU, Y., WENDLER, F., DASGUPTA, R. et al., (2006). Functional genomics reveals genes involved in protein secretion and Golgi organization. Nature 439: 604-607.

BOUGHNER, L.A. and DOERRLER, W.T. (2012). Multiple deletions reveal the essentiality of the DedA membrane protein family in Escherichia coli. Microbiology 
158: 1162-1171.

CALVO-GARRIDO, J., CARILLA-LATORRE, S. and ESCALANTE, R. (2008a). Vacuole membrane protein 1, autophagy and much more. Autophagy $4: 835-837$.

CALVO-GARRIDO, J., CARILLA-LATORRE, S., LA, F., EGEA, G. and ESCALANTE, R. (2008b). Vacuole Membrane Protein 1 Is an Endoplasmic Reticulum Protein Required for Organelle Biogenesis, Protein Secretion, and Development. Mol. Biol. Cell. 19: 3442-3453.

CALVO-GARRIDO, J., CARILLA-LATORRE, S., LAZARO-DIEGUEZ, F., EGEA, G. and ESCALANTE, R. (2008c). Vacuole membrane protein 1 is an endoplasmic reticulum protein required for organelle biogenesis, protein secretion, and development. Mol Biol Cell 19: 3442-3453.

CALVO-GARRIDO, J. and ESCALANTE, R. (2010). Autophagy dysfunction and ubiquitin-positive protein aggregates in Dictyostelium cells lacking Vmp1. Autophagy 6: 100-109.

CALVO-GARRIDO, J., KING, J.S., MUNOZ-BRACERAS, S. and ESCALANTE, R. (2014). Vmp1 Regulates Ptdlns3P Signaling During Autophagosome Formation in Dictyostelium discoideum. Traffic 15: 1235-1246.

DOERRLER, W.T., SIKDAR, R., KUMAR, S. and BOUGHNER, L.A. (2013). New functions for the ancient DedA membrane protein family. J Bacteriol 195: 3-11.

INADOME, H., NODA, Y., ADACHI, H. and YODA, K. (2005). Immunoisolaton of the yeast Golgi subcompartments and characterization of a novel membrane protein, Svp26, discovered in the Sed5-containing compartments. Mol Cell Biol 25: 7696-7710.

KELLER, R., SCHLEPPI, N., WEIKUM, J. and SCHNEIDER, D. (2015). Mutational analyses of YqjA, a Tvp38/DedA protein of E. coli. FEBS Lett 589: 842-848.

KELLER, R. and SCHNEIDER, D. (2013). Homologs of the yeast Tvp38 vesicleassociated protein are conserved in chloroplasts and cyanobacteria. Front. Plant Sci. 4: 467

KUMAR, S. and DOERRLER, W.T. (2014). Members of the conserved DedAfamily are likely membrane transporters and are required for drug resistance in Escherichia coli. Antimicrob Agents Chemother. 58: 923-30.

KUMAR, S. and DOERRLER, W.T. (2015). Escherichia coli YqjA, a Member of the Conserved DedA/Tvp38 Membrane Protein Family, Is a Putative Osmosensing Transporter Required for Growth at Alkaline pH. J Bacteriol 197: 2292-2300.

LEDGHAM, F., QUEST, B., VALLAEYS, T., MERGEAY, M. and COVES, J. (2005). A probable link between the DedA protein and resistance to selenite. Res. Microbiol. 156: 367-374.

LIANG, F.T., XU, Q., SIKDAR, R., XIAO, Y., COX, J.S. and DOERRLER, W.T. (2010) BB0250 of Borrelia burgdorferi is a conserved and essential inner membrane protein required for cell division. J Bacteriol 192: 6105-6115.

LIMA, W.C., PILLONEL, T., BERTELLI, C., IFRID, E., GREUB, G. and COSSON, P. (2018). Genome sequencing and functional characterization of the non-pathogenic Klebsiella pneumoniae KpGe bacteria. Microbes and infection 20: 293-301.
MORETTI, F., BERGMAN, P., DODGSON, S., MARCELLIN, D., CLAERR, I., GOOD WIN, J.M., DEJESUS, R., KANG, Z., ANTCZAK, C., BEGUE, D. et al., (2018). TMEM41B is a novel regulator of autophagy and lipid mobilization. EMBO Rep 19. Epub 2018 Aug 20.

MORITA, K., HAMA, Y., IZUME, T., TAMURA, N., UENO, T., YAMASHITA, Y., SAKAMAKI, Y., MIMURA, K., MORISHITA, H., SHIHOYA, W. et al., (2018). Genomewide CRISPR screen identifies TMEM41B as a gene required for autophagosome formation. J. Cell Biol. 217: 3817-3828

PANG, K.M., LYNES, M.A. and KNECHT, D.A. (1999). Variables controlling the expression level of exogenous genes in Dictyostelium. Plasmid 41: 187-197.

SIKDAR, R. and DOERRLER, W.T. (2010). Inefficient Tat-dependent export of periplasmic amidases in an Escherichia coli strain with mutations in two DedA family genes. J Bacteriol 192: 807-818.

SIKDAR, R., SIMMONS, A.R. and DOERRLER, W.T. (2013). Multiple envelope stress response pathways are activated in an Escherichia coli strain with mutations in two members of the DedA membrane protein family. J Bacteriol 195: 12-24.

TABARA, L.C. and ESCALANTE, R. (2016). VMP1 Establishes ER-Microdomains that Regulate Membrane Contact Sites and Autophagy. PLoS ONE 11: e0166499.

TABARA, L.C., VICENTE, J.J., BIAZIK, J., ESKELINEN, E.L., VINCENT, O. and ESCALANTE, R. (2018). Vacuole membrane protein 1 marks endoplasmic reticulum subdomains enriched in phospholipid synthesizing enzymes and is required for phosphoinositide distribution. Traffic 19: 624-638.

TENENBOIM, H., SMIRNOVA, J., WILLMITZER, L., STEUP, M. and BROTMAN, Y (2014). VMP1-deficient Chlamydomonas exhibits severely aberrant cell morphology and disrupted cytokinesis. BMC Plant Biol 14: 121

THOMPKINS, K., CHATTOPADHYAY, B., XIAO, Y., HENK, M.C. and DOERRLER W.T. (2008). Temperature sensitivity and cell division defects in an Escherichia coli strain with mutations in yghB and yqj $A$, encoding related and conserved inner membrane proteins. J Bacteriol 190: 4489-4500.

TIAN, Y., LI, Z., HU, W., REN, H., TIAN, E., ZHAO, Y., LU, Q., HUANG, X., YANG P., LI, X. et al., (2010). C. elegans screen identifies autophagy genes specific to multicellular organisms. Cell 141: 1042-1055

VELTMAN, D.M., AKAR, G., BOSGRAAF, L. and VAN HAASTERT, P.J. (2009). Anew set of small, extrachromosomal expression vectors for Dictyostelium discoideum. Plasmid 61: 110-118

WANG, P., HUMMEL, E., OSTERRIEDER, A., MEYER, A.J., FRIGERIO, L., SPARKES I. and HAWES, C. (2011). KMS1 and KMS2, two plant endoplasmic reticulum proteins involved in the early secretory pathway. Plant J. 66: 613-28.

ZHAO, Y.G., CHEN, Y., MIAO, G., ZHAO, H., QU, W., LI, D., WANG, Z., LIU, N., LI, L., CHEN, S. et al., (2017). The ER-Localized Transmembrane Protein EPG-3/ VMP1 Regulates SERCAActivity to Control ER-Isolation Membrane Contacts for Autophagosome Formation. Molecular Cell 67: 974-989 e6. 


\section{Further Related Reading, published previously in the Int. J. Dev. Biol.}

YelA, a putative Dictyostelium translational regulator, acts as antagonist of DIF-1 signaling to control cell-type proportioning Yoko Yamada, Chris Sugden and Jeffrey G. Williams

Int. J. Dev. Biol. (2017) 61: 35-42

https://doi.org/10.1387/ijdb.160160yy

Bimodal distribution of motility and cell fate in Dictyostelium discoideum

Pavana Goury-Sistla, Vidyanand Nanjundiah and Gopal Pande

Int. J. Dev. Biol. (2012) 56: 263-272

https://doi.org/10.1387/ijdb.113384ps

Synergy between two transcription factors directs gene expression in Dictyostelium tip-organiser cells Hong Yu Wang and Jeffrey G. Williams

Int. J. Dev. Biol. (2010) 54: 1301-1307

https://doi.org/10.1387/ijdb.103141hw

Pattern formation mechanisms in reaction-diffusion systems

Vladimir K. Vanag and Irving R. Epstein

Int. J. Dev. Biol. (2009) 53: 673-681

https://doi.org/10.1387/ijdb.072484vv

Identification of new modes of Dd-STATa regulation of gene expression in Dictyostelium by in situ hybridisation

Nao Shimada, Mineko Maeda, Hideko Urushihara and Takefumi Kawata

Int. J. Dev. Biol. (2004) 48: 679-682

http://www.intjdevbiol.com/web/paper/041862ns

Cell cycle phase, cellular Ca2+ and development in Dictyostelium discoideum.

MAzhar, P K Kennady, G Pande, M Espiritu, WHolloman, D Brazill, RH Gomer and V Nanjundiah Int. J. Dev. Biol. (2001) 45: 405-414

http://www.intjdevbiol.com/web/paper/11330860

Development at the edge of multi-cellularity: Dictyostelium discoideum

R R Kay

Int. J. Dev. Biol. (2000) 44: 35-38

http://www.intjdevbiol.com/web/paper/10761844

Regulation of cell differentiation and pattern formation in Dictyostelium development I Takeuchi, M Tasaka, K Okamoto and Y Maeda Int. J. Dev. Biol. (1994) 38: 311-319 http://www.intjdevbiol.com/web/paper/7981039

5 yr ISI Impact Factor $(2016)=2.421$
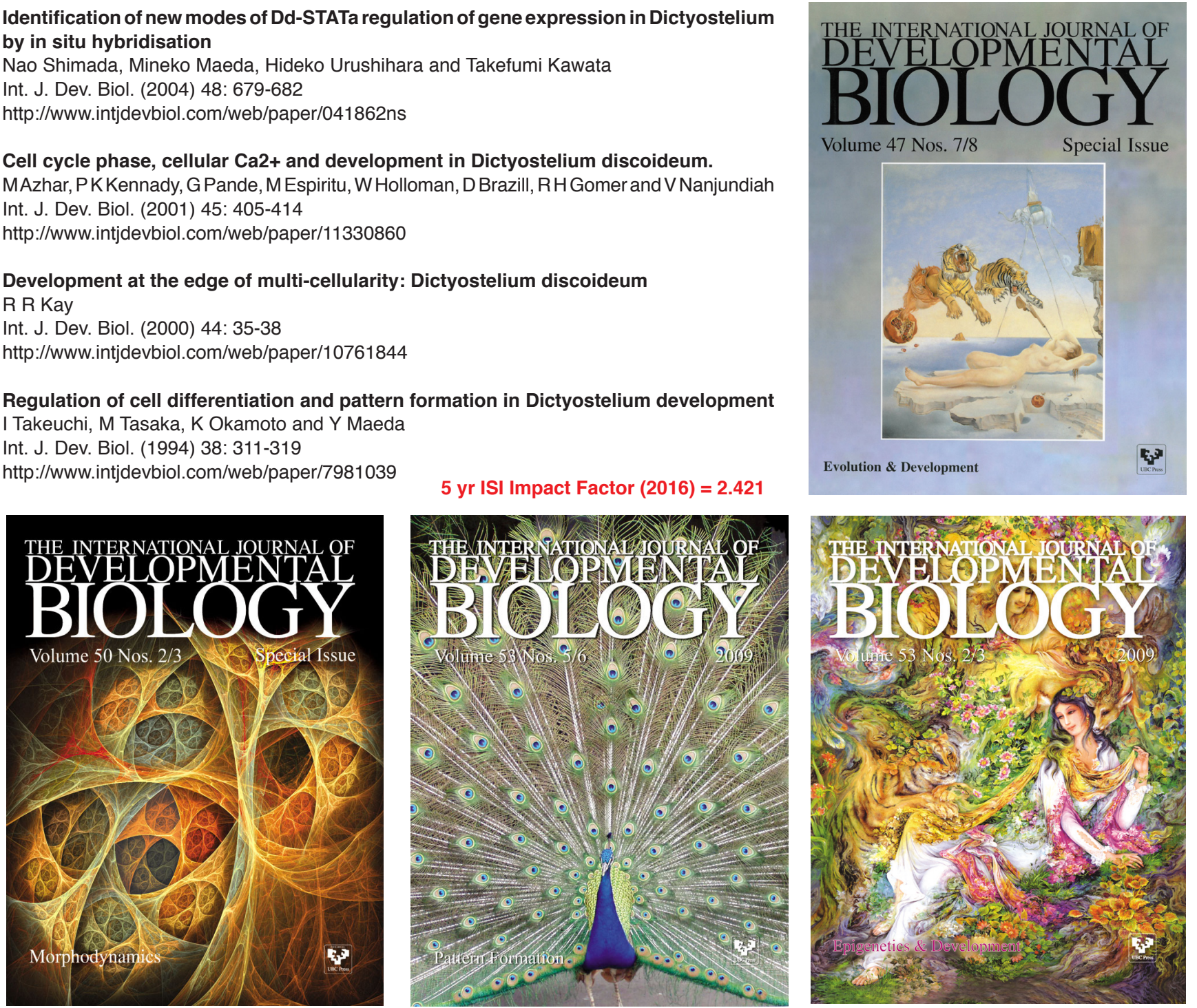